\title{
Allylic Alkylation versus Michael induced Ring Closure - Chelated Enolates as versatile Nucleophiles
}

\author{
Matthias Pohlman, Uli Kazmaier, * Thomas Lindner \\ Institut für Organische Chemie, Universität des Saarlandes, D-66123 Saarbrücken, Germany. \\ E-mail: u.kazmaier@mx.uni-saarland.de
}

\section{Table of contents}

\section{Preparation and Analytical / Spectroscopic Data of Substrates and Products}

Methyl 4-methoxycarbonyloxy-2-pentinoate (8a') $\quad$ S2

Methyl 4-methoxycarbonyloxy-2(Z)-pentenoate (8a) S2

Methyl 4-methoxycarbonyloxy-2-butinoate (8b') S3

Methyl 4-methoxycarbonyloxy-2(Z)-butenoate $((\boldsymbol{Z})-\mathbf{8 b}) \quad$ S3

Methyl 4-methoxycarbonyloxy-2(E)-butenoate $((\boldsymbol{E})-\mathbf{8 b}) \quad$ S3

Methyl 4-tert.-butyloxycarbonyloxy-2-pentinoate (8c') S3

Methyl 4-tert.-butyloxycarbonyloxy-2(Z)-pentenoate (8c) S4

Dimethyl 2-methoxycarbonyl-3-methyl-4(E)-hexendioate (13) S4

Methyl 5-methoxycarbonyloxy-2-pentinoate (16') S4

Methyl 5-methoxycarbonyloxy-2(Z)-pentenoate (16) S4

3-[tert.-Butyloxycarbonyl-(trifluoroacetylamino)-methyl]- $\delta$-valerolactone (17) $\quad$ S5

XRay-Data 15 acid $\quad$ S6

$\begin{array}{ll}\text { Cif-File } 15 \text { acid } & \text { S10 }\end{array}$ 


\section{$\underline{\text { Supporting Informations }}$}

\section{Preparation and Analytical / Spectroscopic Data of Substrates and Products}

The allylic substrates $\mathbf{8}$ were obtained according to:

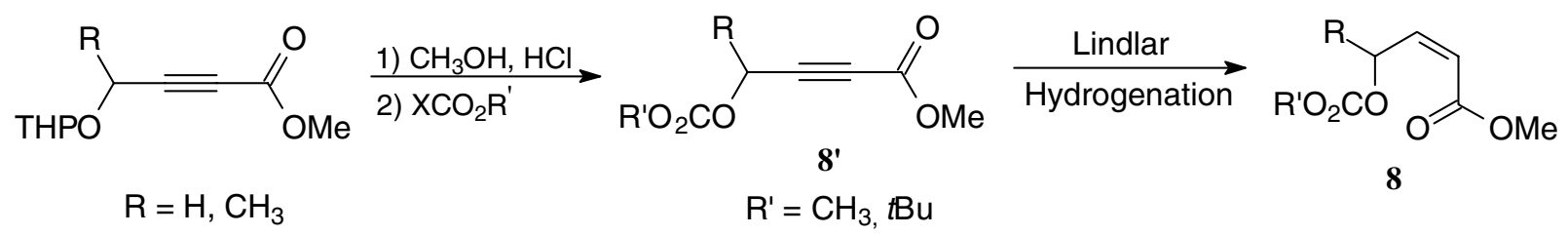

Methyl 4-methoxycarbonyloxy-2-pentinoate (8a'). Two drops of conc. $\mathrm{HCl}$ solution were added to a solution of methyl 4-tetrahydropyranyloxy-2-pentinoate ${ }^{1}(2.02 \mathrm{~g}, 9.53 \mathrm{mmol})$ in methanol (20 $\mathrm{mL})$. After complete consumption of this THP-ether the reaction was quenched by addition of solid $\mathrm{NaHCO}_{3}(1 \mathrm{~g})$ and the solvent was removed in vacuo. The residue was dissolved in $\mathrm{H}_{2} \mathrm{O} / \mathrm{CH}_{2} \mathrm{Cl}_{2}(20$ $\mathrm{mL}$ each), the layers were separated and the aqueous layer was extracted 5 times with $\mathrm{CH}_{2} \mathrm{Cl}_{2}(20$ $\mathrm{mL}$ each). The combined organic layers were dried $\left(\mathrm{Na}_{2} \mathrm{SO}_{4}\right)$ and the solvent was evaporated in vacuo. The crude product was dissolved in $\mathrm{CH}_{2} \mathrm{Cl}_{2}(20 \mathrm{~mL})$ and the solution was cooled to $0^{\circ} \mathrm{C}$ before pyridine $(2 \mathrm{~mL}, 25 \mathrm{mmol})$ and methyl chloroformiate $(1.55 \mathrm{~mL}, 20 \mathrm{mmol})$ were added. After complete consumption of the alcohol (TLC) the mixture was hydrolyzed with $1 \mathrm{~N} \mathrm{KHSO}_{4}(30 \mathrm{~mL})$. The layers were separated and the aqueous layer was extracted twice with $\mathrm{CH}_{2} \mathrm{Cl}_{2}$. The combined organic layer was dried $\left(\mathrm{Na}_{2} \mathrm{SO}_{4}\right)$ and the solvent was removed in vacuo. The crude product was purified by flash chromatography (hexanes / EtOAc $9: 1)$ giving rise to 8a' (1.59 g, 8.54 mmol, $90 \%)$ as a colorless liquid. ${ }^{1} \mathrm{H}$ NMR $(300 \mathrm{MHz}): \delta=1.57(\mathrm{~d}, J=6.9 \mathrm{~Hz}, 3 \mathrm{H}), 3.76,3.80(2 \mathrm{~s}, 6 \mathrm{H})$, $5.38(\mathrm{q}, J=6.9 \mathrm{~Hz}, 1 \mathrm{H}) .{ }^{13} \mathrm{C} \mathrm{NMR}(75 \mathrm{MHz}): \delta=20.3,52.8,55.2,63.3,76.6,84.2,153.3,154.5$.

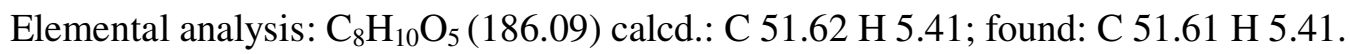

Methyl 4-methoxycarbonyloxy-2(Z)-pentenoate (8a). Alkinoate (8a') (618 mg, $3.32 \mathrm{mmol}$ ) was dissolved in a mixture of hexanes $(9 \mathrm{~mL})$ and tert.-butanol $(2.2 \mathrm{~mL})$ before $5 \% \mathrm{Pd} / \mathrm{BaSO}_{4}(50 \mathrm{mg})$ and quinoline $(75 \mathrm{mg}$ ) were added. The flask was evacuated and flushed with hydrogen from a hydrogen burette, which was used to measure the consumption of hydrogen. The reaction mixture was stirred vigorously and the hydrogenation was stopped after the consumption of 1 eq. of $\mathrm{H}_{2}$. After flash chromatography 8a' was obtained as a colorless liquid (550 mg, $2.93 \mathrm{mmol}, 88 \%$ ).

\footnotetext{
${ }^{1}$ Cornelius, L. A. M.; Bone, R. G. A.; Hastings, R. H.; Deardoff, M. A.; Scharlach, R. A.; Hauptmann, B. E.; Stankovic, C. S.; Pinnick, H. W. J. Org. Chem. 1993, 58, 3188-3190.
} 
${ }^{1} \mathrm{H}$ NMR (300 MHz): $\delta=1.40(\mathrm{~d}, J=6.3 \mathrm{~Hz}, 3 \mathrm{H}), 3.71(\mathrm{~s}, 3 \mathrm{H}), 3.74(\mathrm{~s}, 3 \mathrm{H}), 5.80(\mathrm{~d}, J=10.3 \mathrm{~Hz}$, 1H), 6.10-6.23 (m, 2H). ${ }^{13} \mathrm{C}$ NMR (75 MHz): $\delta=19.5,51.5,54.6,72.2,119.7,147.8,155.0,165.7$. Elemental analysis: $\mathrm{C}_{8} \mathrm{H}_{12} \mathrm{O}_{5}$ (188.09) calcd.: C $51.06 \mathrm{H}$ 6.43; found: C $51.13 \mathrm{H} 6.56$.

Methyl 4-methoxycarbonyloxy-2-butinoate (8b'). According to 8a' 8b' was obtained from methyl 4-tetrahydropyranyloxy-2-butinoate $(817 \mathrm{mg}, 4.12 \mathrm{mmol})$ after flash chromatography (hexanes / ether $8: 2)$ and bulb to bulb distillation $\left(\mathrm{kp}_{0.03}: 75^{\circ} \mathrm{C}\right)$ as colorless liquid. Yield: $391 \mathrm{mg}(2.27 \mathrm{mmol}$, $55 \%) .{ }^{1} \mathrm{H}$ NMR (300 MHz): $\delta=3.76,3.80(2 \mathrm{~s}, 6 \mathrm{H}), 4.82(\mathrm{~s}, 2 \mathrm{H}) .{ }^{13} \mathrm{C} \mathrm{NMR}(75 \mathrm{MHz}): \delta=52.9$, 55.4, 54.6, 78.3, 80.4, 153.1, 154.9. Elemental analysis: $\mathrm{C}_{7} \mathrm{H}_{8} \mathrm{O}_{5}(172,14)$ calcd.: $\mathrm{C} 48.84 \mathrm{H} 4.68$; found: C $48.82 \mathrm{H} 4.92$.

Methyl 4-methoxycarbonyloxy-2(Z)-butenoate ((Z)-8b). According to 8a $(Z)-8 b$ was obtained from 8b' (800 mg , $4.64 \mathrm{mmol}$ ) after flash chromatography (hexanes / EtOAc $88: 12$ ) as a colorless liquid. Yield: $802 \mathrm{mg}$ (4.60 mmol, 99\%). ${ }^{1} \mathrm{H}$ NMR (300 MHz): $\delta=3.71$ (s, 3H), 3.78 (s, 3H), 5.23 $(\mathrm{dd}, J=4.8 \mathrm{~Hz}, J=2.2 \mathrm{~Hz}, 2 \mathrm{H}), 5.86(\mathrm{dt}, J=11.8 \mathrm{~Hz}, J=2.4 \mathrm{~Hz}, 1 \mathrm{H}), 6.27(\mathrm{dt}, J=11.4 \mathrm{~Hz}, J=5.0$ $\mathrm{Hz}, 1 \mathrm{H}) .{ }^{13} \mathrm{C}$ NMR (125 MHz): $\delta=51.5$ (q, C-1), 54.9 (q, C-7), 66.0 (t, C-5), 120.4 (d, C-3), 144.2 (d, C-4), 155.5 (s, C-6), 166.0 (s, C-2). Elemental analysis: $\mathrm{C}_{7} \mathrm{H}_{10} \mathrm{O}_{5}$ (174.15) calcd.: C 48.28 H 5.79; found: C $48.11 \mathrm{H} 5.93$.

Methyl 4-methoxycarbonyloxy-2(E)-butenoate ((E)-8b). According to 8a' $(E)-8 b$ was obtained from methyl 4-hydroxy-2(E)-butenoate (235 mg, $2.03 \mathrm{mmol}$ ) in 92\% yield (325 mg, $1.87 \mathrm{mmol}$ ) after flash chromatography (hexanes / EtOAc $85: 15) .{ }^{1} \mathrm{H}$ NMR $(300 \mathrm{MHz}): \delta=3.72(\mathrm{~s}, 3 \mathrm{H}), 3.79$ (s, 3H), $4.77(\mathrm{dd}, J=4.6 \mathrm{~Hz}, J=2.0 \mathrm{~Hz}, 2 \mathrm{H}), 6.04$ (dt, $J=15.8 \mathrm{~Hz}, J=1.9 \mathrm{~Hz}, 1 \mathrm{H}), 6.91(\mathrm{dt}, J=15.8$ $\mathrm{Hz}, J=4.5 \mathrm{~Hz}, 1 \mathrm{H}) .{ }^{13} \mathrm{C} \mathrm{NMR}(75 \mathrm{MHz}): \delta=51.7,55.0,65.8,122.1,140.6,155.2,166.1$. Elemental analysis: $\mathrm{C}_{7} \mathrm{H}_{10} \mathrm{O}_{5}(174.15)$ calcd.: C 48.28 H 5.79; found: $\mathrm{C} 47.91 \mathrm{H} 5.90$.

Methyl 4-tert.-butyloxycarbonyloxy-2-pentinoate (8c'). According to 8a' 8c' was obtained from 4-tetrahydropyranyloxy-2-pentinoate $(450 \mathrm{mg}, 2.12 \mathrm{mmol})$. Di-tert.-butyl dicarbonate $(650 \mathrm{mg}, 3$ mmol), $\mathrm{NEt}_{3}(1 \mathrm{~mL})$ and DMAP $(50 \mathrm{mg}, 0.4 \mathrm{mmol})$ were used for carbonate formation. The crude product was purified by flash chromatography (hexanes / EtOAc $98: 2$ ) and bulb to bulb distillation $\left(\mathrm{Kp}_{0.06}: 130^{\circ} \mathrm{C}\right)$ giving rise to $\mathbf{8 c}{ }^{\prime}(205 \mathrm{mg}, 0.90 \mathrm{mmol}, 42 \%)$ as a colorless liquid. ${ }^{1} \mathrm{H}$ NMR (500 $\mathrm{MHz}): \delta=1.42(\mathrm{~s}, 9 \mathrm{H}), 1.49(\mathrm{~d}, J=6,9 \mathrm{~Hz}, 3 \mathrm{H}), 3.69(\mathrm{~s}, 3 \mathrm{H}), 5.27$ (q, $J=6.8 \mathrm{~Hz}, 1 \mathrm{H}) .{ }^{13} \mathrm{C} \mathrm{NMR}$ (125 MHz): $\delta=20.4,27.7,52.8,62.2,76.3,83.3,84.8,152.1,153.4$. Elemental analysis: $\mathrm{C}_{11} \mathrm{H}_{16} \mathrm{O}_{5}$ (228.25) calcd.: C 57.89 H 7.07; found: C 58.11 H 7.07. 
Methyl 4-tert.-butyloxycarbonyloxy-2(Z)-pentenoate (8c). According to 8a 8c was obtained from 8c' (205 mg, $0.90 \mathrm{mmol}$ ) after flash chromatography (hexanes / EtOAc $97: 3$ ) as a colorless liquid. Yield: $144 \mathrm{mg}$ (0.63 mmol, 70\%). ${ }^{1} \mathrm{H}$ NMR (500 MHz): $\delta=1.39$ (d, $\left.J=6.4 \mathrm{~Hz}, 3 \mathrm{H}\right), 1.45$ (s, 9H), $3.71(\mathrm{~s}, 3 \mathrm{H}), 5.78(\mathrm{~d}, J=11,0 \mathrm{~Hz}, 1 \mathrm{H}), 6.10(\mathrm{~m}, 1 \mathrm{H}), 6.18(\mathrm{dd}, J=11.9 \mathrm{~Hz}, J=7.3 \mathrm{~Hz}, 1 \mathrm{H}) .{ }^{13} \mathrm{C}$ NMR (125 MHz): $\delta=19.6,27.9,51.5,76.7,82.3,119.3,148.8,152.7,165.8$. HMRS (EI): calcd for $\mathrm{C}_{11} \mathrm{H}_{19} \mathrm{O}_{5}\left([\mathrm{M}+\mathrm{H}]^{+}\right), 231,1231$; found, 231,1232.

Dimethyl 2-methoxycarbonyl-3-methyl-4(E)-hexendioate (13). Triphenylphosphine (10 mg, 37.7 $\mu \mathrm{mol})$ and allyl palladiumchloride dimer $(2 \mathrm{mg}, 5.0 \mu \mathrm{mol})$ were dissolved in THF $(1 \mathrm{~mL})$. After stirring at room temperature. for $30 \mathrm{~min}$ a solution of $\mathbf{8 a}(98 \mathrm{mg}, 0.52 \mathrm{mmol})$ and dimethylmalonate (103 mg, $0.78 \mathrm{mmol}$ ) was added. The solution was stirred overnight at rt. The solvent was removed in vacuo and the crude product was purified by flash chromatography. 13 was obtained as colorless oil in 80\% yield (101 mg, $0.42 \mathrm{mmol}) .{ }^{1} \mathrm{H}$ NMR (300 MHz): $\delta=1.12(\mathrm{~d}, J=7.0 \mathrm{~Hz}, 3 \mathrm{H}), 3.09$ (m, 1H), 3.36 (d, $J=8.8 \mathrm{~Hz}, 1 \mathrm{H}), 3.68,3.69,3.72(3 \mathrm{~s}, 9 \mathrm{H}), 5.84(\mathrm{dd}, J=15.8 \mathrm{~Hz}, J=1.1 \mathrm{~Hz}, 1), 6.86$ $(\mathrm{dd}, J=15.4 \mathrm{~Hz}, J=8.1 \mathrm{~Hz}, 1 \mathrm{H}) .{ }^{13} \mathrm{C} \mathrm{NMR}(75 \mathrm{MHz}): \delta=17.3,36.3,51.5,52.5,52.6,56.6,121.7$, 149.1, 168.0, 168.1, 168.6. Elemental analysis: $\mathrm{C}_{11} \mathrm{H}_{16} \mathrm{O}_{6}(244.24)$ calcd.: C 54.10 H 6.60; found: C 54.26 H 6.58 .

Methyl 5-methoxycarbonyloxy-2-pentinoate (16'). A solution of 3-butin-1-ol (1.01 g, $14.4 \mathrm{mmol})$ in abs. THF ( $35 \mathrm{~mL})$ was cooled to $-78^{\circ} \mathrm{C}$. During $10 \mathrm{~min} 18 \mathrm{~mL}(28.8 \mathrm{mmol})$ of $1.6 \mathrm{M} \mathrm{BuLi}$ was added, and the mixture was stirred for $30 \mathrm{~min}$ at this temperature before the cooling bath was removed. During warmup a white precipitate was formed and the suspension was cooled again to $78^{\circ} \mathrm{C}$. Methyl chloroformiate $(2.35 \mathrm{~mL}, 30.2 \mathrm{mmol})$ was added dropwise during $5 \mathrm{~min}$. After warming to room temperature overnight, the solution was diluted with ether before sat. $\mathrm{NH}_{4} \mathrm{Cl}$ was added. The layers were separated and the aqueous layer was extracted four times with $\mathrm{CH}_{2} \mathrm{Cl}_{2}$. The combined organic layers were dried $\left(\mathrm{Na}_{2} \mathrm{SO}_{4}\right)$ and the solvent was evaporated in vacuo. The crude product was purified by bulb to bulb distillation $\left(\mathrm{Kp}_{0.02}: 150^{\circ} \mathrm{C}\right)$ giving rise to $16^{\prime}$ as a colorless liquid. Yield: $1.80 \mathrm{~g}(9.70 \mathrm{mmol}, 67 \%) .{ }^{1} \mathrm{H}$ NMR (500 MHz): $\delta=2.70(\mathrm{t}, J=6.6 \mathrm{~Hz}, 2 \mathrm{H}), 3.77,3.73$ $(2 \mathrm{~s}, 6 \mathrm{H}), 4.24(\mathrm{t}, J=6.8 \mathrm{~Hz}, 2 \mathrm{H}) .{ }^{13} \mathrm{C} \mathrm{NMR}(125 \mathrm{MHz}): \delta=19.2,52.6,55.0,64.2,74.2,84.1,153.5$, 155.3. Elemental analysis: $\mathrm{C}_{8} \mathrm{H}_{10} \mathrm{O}_{5}$ (186.16) calcd.: C 51.61 H 5.41; found: C 51.59 H 5.51.

Methyl 5-methoxycarbonyloxy-2(Z)-pentenoate (16). According to 8a' 16' (700 mg, 3.75 mmol) was hydrogenated using $\mathrm{Pd} / \mathrm{CaCO}_{3}(20 \mathrm{mg})$ and quinoline $(100 \mathrm{mg})$ over a period of $26 \mathrm{~h}$. The 
crude product was purified by flash chromatography (hexanes / EtOAc 97 : 3). Yield: $630 \mathrm{mg}$ (3.34 mmol, $89 \%) 16$ as colorless liquid. ${ }^{1} \mathrm{H}$ NMR (500 MHz): $\delta=3.02(\mathrm{~m}, 2 \mathrm{H}), 3.69,3.75(2 \mathrm{~s}, 6 \mathrm{H}), 4.22$ $(\mathrm{t}, J=6.3 \mathrm{~Hz}, 2 \mathrm{H}), 5.87(\mathrm{~d}, J=11.4 \mathrm{~Hz}, 1 \mathrm{H}), 6.24(\mathrm{dt}, J=11.7 \mathrm{~Hz}, J=7.3 \mathrm{~Hz}, 1 \mathrm{H}) .{ }^{13} \mathrm{C}$ NMR $(125$ MHz): $\delta=28.4,51.1,54.7,66.7,121.8,144.6,155.7,166.4$. Elemental analysis: $\mathrm{C}_{8} \mathrm{H}_{12} \mathrm{O}_{5}(188.09)$ calcd.: C 51.06 H 6.43; found: C 51.19 H 6.46.

\section{3-[tert.-Butyloxycarbonyl-(trifluoroacetylamino)-methyl]- $\delta$-valerolactone (17). According to} 15 lactone 17 was obtained from 16 (197 mg, $1.05 \mathrm{mmol})$ in 85\% yield (342 $\mathrm{mg}, 0.89 \mathrm{mmol}$ ) as white solid, mp. $108-110^{\circ} \mathrm{C}$. Diastereomeric ratio: 93/7. ${ }^{1} \mathrm{H}$ NMR $(500 \mathrm{MHz}): \delta=1.48(\mathrm{~s}, 9 \mathrm{H})$, $1.72-1.83(\mathrm{~m}, 1 \mathrm{H}), 2,03-2,12(\mathrm{~m}, 1 \mathrm{H}), 3,03(\mathrm{~m}, 1 \mathrm{H}), 3.60(\mathrm{~d}, J=8.8 \mathrm{~Hz}, 1 \mathrm{H}), 3.79(\mathrm{~s}, 3 \mathrm{H}), 4.28$ $(\mathrm{ddd}, J=J=10.7 \mathrm{~Hz}, J=3.1 \mathrm{~Hz}, 1 \mathrm{H}), 4.36(\mathrm{ddd}, J=11.5 \mathrm{~Hz}, J=J=4.4 \mathrm{~Hz}, 1 \mathrm{H}), 4.50(\mathrm{dd}, J=7.3$ $\mathrm{Hz}, J=5.4 \mathrm{~Hz}, 1 \mathrm{H}), 7.10(\mathrm{~d}, J=6.9 \mathrm{~Hz}, 1 \mathrm{H}) .{ }^{13} \mathrm{C} \mathrm{NMR}(125 \mathrm{MHz}): \delta=25.0,27.9,37.2,49.7,53.2$, 55.6, 67.2, 85.3, 115.5 (q, $J=287.7 \mathrm{~Hz}), 157.3(\mathrm{q}, J=37.9 \mathrm{H}), 166.2$, 167.6, 168.6. Selected signals of the syn-diastereomer: ${ }^{1} \mathrm{H}$ NMR $(500 \mathrm{MHz}): \delta=3.72(\mathrm{~d}, J=7.1 \mathrm{~Hz}, 1 \mathrm{H}), 3.77(\mathrm{~s}, 3 \mathrm{H}), 4.78(\mathrm{dd}, J=$ $9.7 \mathrm{~Hz}, J=4.9 \mathrm{~Hz}, 1 \mathrm{H}) .{ }^{13} \mathrm{C} \mathrm{NMR}(125 \mathrm{MHz}): \delta=24.3$, 27.8, 35.3, 47.9, 67.4, 84.0. Elemental analysis: $\mathrm{C}_{15} \mathrm{H}_{20} \mathrm{~F}_{3} \mathrm{NO}_{7}(383.32)$ calcd.: C $47.00 \mathrm{H} 5.26 \mathrm{~N} 3.65$; found: C $46.91 \mathrm{H} 5.28 \mathrm{~N} 3.75$. 

acid)

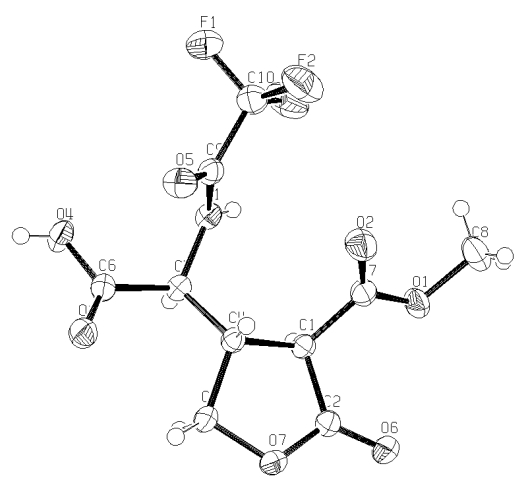

Table 1. Crystal data and structure refinement for $\mathbf{1 5}$ acid.

Identification code

Empirical formula

Formula weight

Temperature

Wavelength

Crystal system

Space group

Unit cell dimensions

Volume

$\mathrm{Z}$

Density (calculated)

Absorption coefficient

$\mathrm{F}(000)$

Crystal size

Theta range for data collection

Index ranges

Reflections collected

Independent reflections

Completeness to theta $=24.04^{\circ}$

Absorption correction

Refinement method

Data / restraints / parameters

Goodness-of-fit on $\mathrm{F}^{2}$

Final $\mathrm{R}$ indices [I $>2 \operatorname{sigma}(\mathrm{I})]$

$\mathrm{R}$ indices (all data)

Largest diff. peak and hole shelxs 1843

C10 H10 F3 N O7

313.19

293(2) K

$0.71073 \AA$

Triclinic

P-1

$\mathrm{a}=5.1390(10) \AA \quad \alpha=98.61(3)^{\circ}$.

$\mathrm{b}=7.3910(10) \AA \quad \beta=96.12(3)^{\circ}$.

$\mathrm{c}=16.684(3) \AA \quad \gamma=94.06(3)^{\circ}$.

$620.55(18) \AA^{3}$

2

$1.676 \mathrm{Mg} / \mathrm{m}^{3}$

$0.169 \mathrm{~mm}^{-1}$

320

$0.3 \times 0.25 \times 0.2 \mathrm{~mm}^{3}$

2.80 to $24.04^{\circ}$.

$-5<=\mathrm{h}<=5,-8<=\mathrm{k}<=8,-17<=\mathrm{l}<=18$

3718

$1729[\mathrm{R}(\mathrm{int})=0.0854]$

$88.5 \%$

N/A

Full-matrix least-squares on $\mathrm{F}^{2}$

1729 / 0 / 226

1.048

$\mathrm{R} 1=0.0529, \mathrm{wR} 2=0.1465$

$\mathrm{R} 1=0.0583, \mathrm{wR} 2=0.1511$

0.288 and -0.261 e. $\AA^{-3}$ 
Table 2. Atomic coordinates ( $\mathrm{x} 10^{4}$ ) and equivalent isotropic displacement parameters $\left(\AA^{2} \mathrm{x} 10^{3}\right)$ for $\mathbf{1 5}$ acid. $\mathrm{U}(\mathrm{eq})$ is defined as one third of the trace of the orthogonalized Uij tensor.

\begin{tabular}{|c|c|c|c|c|}
\hline & $\mathrm{x}$ & $\mathrm{y}$ & $\mathrm{z}$ & $\mathrm{U}(\mathrm{eq})$ \\
\hline $\mathrm{O}(1)$ & $-272(4)$ & 1394(3) & $2803(1)$ & $34(1)$ \\
\hline $\mathrm{O}(2)$ & $3784(4)$ & $639(3)$ & $3176(1)$ & $38(1)$ \\
\hline $\mathrm{O}(3)$ & $7293(4)$ & $-4372(3)$ & $1051(1)$ & $31(1)$ \\
\hline $\mathrm{O}(4)$ & $6006(4)$ & $-6422(2)$ & $1839(1)$ & $33(1)$ \\
\hline $\mathrm{O}(5)$ & $8190(4)$ & $-2902(3)$ & 2993(1) & $32(1)$ \\
\hline $\mathrm{O}(6)$ & 40(4) & 1933(3) & $1142(1)$ & $35(1)$ \\
\hline $\mathrm{O}(7)$ & $2613(4)$ & $-53(3)$ & $549(1)$ & $32(1)$ \\
\hline $\mathrm{N}(1)$ & $3762(5)$ & $-3552(3)$ & 2731(1) & $24(1)$ \\
\hline $\mathrm{C}(1)$ & $1582(5)$ & $-614(3)$ & $1823(2)$ & $22(1)$ \\
\hline$C(2)$ & $1267(6)$ & $590(4)$ & $1159(2)$ & $26(1)$ \\
\hline$C(3)$ & $3731(6)$ & $-1783(4)$ & $677(2)$ & $30(1)$ \\
\hline $\mathrm{C}(4)$ & $3883(5)$ & $-1729(3)$ & $1604(2)$ & $22(1)$ \\
\hline$C(5)$ & $3712(5)$ & $-3691(4)$ & $1844(2)$ & $22(1)$ \\
\hline$C(6)$ & $5895(5)$ & $-4843(3)$ & $1537(2)$ & $24(1)$ \\
\hline$C(7)$ & $1884(6)$ & 547(4) & $2681(2)$ & $26(1)$ \\
\hline $\mathrm{C}(8)$ & $-287(9)$ & $2489(5)$ & $3614(2)$ & $42(1)$ \\
\hline $\mathrm{C}(9)$ & $6010(6)$ & $-3058(3)$ & $3221(2)$ & $25(1)$ \\
\hline$C(10)$ & $5794(6)$ & $-2720(4)$ & $4144(2)$ & $33(1)$ \\
\hline $\mathrm{F}(1)$ & $6821(5)$ & $-4075(3)$ & $4479(1)$ & $60(1)$ \\
\hline $\mathrm{F}(2)$ & $7117(4)$ & $-1113(3)$ & $4512(1)$ & $54(1)$ \\
\hline $\mathrm{F}(3)$ & $3328(4)$ & $-2696(3)$ & $4317(1)$ & $56(1)$ \\
\hline
\end{tabular}


Table 3. Bond lengths $[\AA]$ and angles $\left[^{\circ}\right]$ for $\mathbf{1 5}$ acid.

\begin{tabular}{|c|c|c|c|}
\hline $\mathrm{O}(1)-\mathrm{C}(7)$ & $1.330(4)$ & $\mathrm{O}(3)-\mathrm{C}(6)-\mathrm{O}(4)$ & $124.4(3)$ \\
\hline $\mathrm{O}(1)-\mathrm{C}(8)$ & $1.470(4)$ & $\mathrm{O}(3)-\mathrm{C}(6)-\mathrm{C}(5)$ & $122.4(2)$ \\
\hline $\mathrm{O}(2)-\mathrm{C}(7)$ & $1.201(4)$ & $\mathrm{O}(4)-\mathrm{C}(6)-\mathrm{C}(5)$ & $113.2(2)$ \\
\hline $\mathrm{O}(3)-\mathrm{C}(6)$ & $1.212(3)$ & $\mathrm{O}(2)-\mathrm{C}(7)-\mathrm{O}(1)$ & $124.8(2)$ \\
\hline $\mathrm{O}(4)-\mathrm{C}(6)$ & $1.341(3)$ & $\mathrm{O}(2)-\mathrm{C}(7)-\mathrm{C}(1)$ & $124.5(3)$ \\
\hline $\mathrm{O}(5)-\mathrm{C}(9)$ & $1.224(3)$ & $\mathrm{O}(1)-\mathrm{C}(7)-\mathrm{C}(1)$ & $110.6(2)$ \\
\hline $\mathrm{O}(6)-\mathrm{C}(2)$ & $1.215(3)$ & $\mathrm{O}(5)-\mathrm{C}(9)-\mathrm{N}(1)$ & $125.2(2)$ \\
\hline $\mathrm{O}(7)-\mathrm{C}(2)$ & $1.339(3)$ & $\mathrm{O}(5)-\mathrm{C}(9)-\mathrm{C}(10)$ & $118.5(2)$ \\
\hline $\mathrm{O}(7)-\mathrm{C}(3)$ & $1.473(3)$ & $\mathrm{N}(1)-\mathrm{C}(9)-\mathrm{C}(10)$ & $116.2(2)$ \\
\hline $\mathrm{N}(1)-\mathrm{C}(9)$ & $1.336(4)$ & $\mathrm{F}(3)-\mathrm{C}(10)-\mathrm{F}(1)$ & $106.9(2)$ \\
\hline $\mathrm{N}(1)-\mathrm{C}(5)$ & $1.465(3)$ & $\mathrm{F}(3)-\mathrm{C}(10)-\mathrm{F}(2)$ & $107.8(2)$ \\
\hline $\mathrm{C}(1)-\mathrm{C}(2)$ & $1.525(4)$ & $\mathrm{F}(1)-\mathrm{C}(10)-\mathrm{F}(2)$ & $108.3(3)$ \\
\hline $\mathrm{C}(1)-\mathrm{C}(4)$ & $1.533(4)$ & $\mathrm{F}(3)-\mathrm{C}(10)-\mathrm{C}(9)$ & $113.2(2)$ \\
\hline $\mathrm{C}(1)-\mathrm{C}(7)$ & $1.541(4)$ & $\mathrm{F}(1)-\mathrm{C}(10)-\mathrm{C}(9)$ & $108.9(2)$ \\
\hline$C(3)-C(4)$ & $1.534(4)$ & $\mathrm{F}(2)-\mathrm{C}(10)-\mathrm{C}(9)$ & $111.5(2)$ \\
\hline$C(4)-C(5)$ & $1.561(4)$ & & \\
\hline$C(5)-C(6)$ & $1.540(4)$ & \multirow{5}{*}{\multicolumn{2}{|c|}{$\begin{array}{l}\text { Symmetry transformations used to } \\
\text { generate equivalent atoms: }\end{array}$}} \\
\hline $\mathrm{C}(9)-\mathrm{C}(10)$ & $1.541(4)$ & & \\
\hline $\mathrm{C}(10)-\mathrm{F}(3)$ & $1.330(4)$ & & \\
\hline $\mathrm{C}(10)-\mathrm{F}(1)$ & $1.332(4)$ & & \\
\hline$C(10)-F(2)$ & $1.352(4)$ & & \\
\hline $\mathrm{C}(7)-\mathrm{O}(1)-\mathrm{C}(8)$ & $116.2(3)$ & & \\
\hline $\mathrm{C}(2)-\mathrm{O}(7)-\mathrm{C}(3)$ & $111.9(2)$ & & \\
\hline $\mathrm{C}(9)-\mathrm{N}(1)-\mathrm{C}(5)$ & $120.4(2)$ & & \\
\hline $\mathrm{C}(2)-\mathrm{C}(1)-\mathrm{C}(4)$ & $103.8(2)$ & & \\
\hline $\mathrm{C}(2)-\mathrm{C}(1)-\mathrm{C}(7)$ & 111.3(2) & & \\
\hline $\mathrm{C}(4)-\mathrm{C}(1)-\mathrm{C}(7)$ & 116.7(2) & & \\
\hline $\mathrm{O}(6)-\mathrm{C}(2)-\mathrm{O}(7)$ & $122.2(2)$ & & \\
\hline $\mathrm{O}(6)-\mathrm{C}(2)-\mathrm{C}(1)$ & $128.8(2)$ & & \\
\hline $\mathrm{O}(7)-\mathrm{C}(2)-\mathrm{C}(1)$ & $109.0(2)$ & & \\
\hline $\mathrm{O}(7)-\mathrm{C}(3)-\mathrm{C}(4)$ & $103.5(2)$ & & \\
\hline $\mathrm{C}(1)-\mathrm{C}(4)-\mathrm{C}(3)$ & $102.4(2)$ & & \\
\hline$C(1)-C(4)-C(5)$ & $114.1(2)$ & & \\
\hline $\mathrm{C}(3)-\mathrm{C}(4)-\mathrm{C}(5)$ & $112.3(2)$ & & \\
\hline $\mathrm{N}(1)-\mathrm{C}(5)-\mathrm{C}(6)$ & $110.8(2)$ & & \\
\hline $\mathrm{N}(1)-\mathrm{C}(5)-\mathrm{C}(4)$ & $109.8(2)$ & & \\
\hline$C(6)-C(5)-C(4)$ & $112.7(2)$ & & \\
\hline
\end{tabular}


Table 4. Anisotropic displacement parameters $\left(\AA^{2} \times 10^{3}\right)$ for $\mathbf{1 5}$ acid. The anisotropic displacement factor exponent takes the form: $-2 \pi^{2}\left[h^{2} a^{* 2} U^{11}+\ldots+2 h k a^{*} b^{*} U^{12}\right]$

\begin{tabular}{lllllll}
\hline & $\mathrm{U}^{11}$ & $\mathrm{U}^{22}$ & $\mathrm{U} 33$ & $\mathrm{U}^{23}$ & $\mathrm{U}$ & $\mathrm{U}$ \\
& & & & & \\
\hline $\mathrm{O}(1)$ & $37(1)$ & $38(1)$ & $28(1)$ & $-1(1)$ & $7(1)$ & $14(1)$ \\
$\mathrm{O}(2)$ & $35(1)$ & $43(1)$ & $33(1)$ & $-2(1)$ & $-4(1)$ & $8(1)$ \\
$\mathrm{O}(3)$ & $32(1)$ & $32(1)$ & $33(1)$ & $9(1)$ & $12(1)$ & $8(1)$ \\
$\mathrm{O}(4)$ & $33(1)$ & $25(1)$ & $44(1)$ & $11(1)$ & $10(1)$ & $10(1)$ \\
$\mathrm{O}(5)$ & $24(1)$ & $42(1)$ & $31(1)$ & $9(1)$ & $4(1)$ & $2(1)$ \\
$\mathrm{O}(6)$ & $43(1)$ & $34(1)$ & $32(1)$ & $10(1)$ & $6(1)$ & $18(1)$ \\
$\mathrm{O}(7)$ & $43(1)$ & $33(1)$ & $27(1)$ & $13(1)$ & $10(1)$ & $16(1)$ \\
$\mathrm{N}(1)$ & $18(1)$ & $26(1)$ & $30(1)$ & $10(1)$ & $8(1)$ & $4(1)$ \\
$\mathrm{C}(1)$ & $21(2)$ & $20(1)$ & $24(1)$ & $5(1)$ & $3(1)$ & $1(1)$ \\
$\mathrm{C}(2)$ & $27(2)$ & $25(1)$ & $25(1)$ & $3(1)$ & $2(1)$ & $4(1)$ \\
$\mathrm{C}(3)$ & $36(2)$ & $28(2)$ & $29(2)$ & $10(1)$ & $9(1)$ & $16(1)$ \\
$\mathrm{C}(4)$ & $21(2)$ & $22(1)$ & $25(1)$ & $6(1)$ & $5(1)$ & $4(1)$ \\
$\mathrm{C}(5)$ & $17(2)$ & $22(1)$ & $27(1)$ & $6(1)$ & $3(1)$ & $0(1)$ \\
$\mathrm{C}(6)$ & $21(2)$ & $22(1)$ & $26(1)$ & $1(1)$ & $0(1)$ & $2(1)$ \\
$\mathrm{C}(7)$ & $30(2)$ & $23(1)$ & $27(2)$ & $5(1)$ & $6(1)$ & $1(1)$ \\
$\mathrm{C}(8)$ & $51(2)$ & $41(2)$ & $32(2)$ & $-6(2)$ & $12(2)$ & $5(2)$ \\
$\mathrm{C}(9)$ & $27(2)$ & $23(1)$ & $27(1)$ & $7(1)$ & $6(1)$ & $4(1)$ \\
$\mathrm{C}(10)$ & $38(2)$ & $33(2)$ & $30(2)$ & $8(1)$ & $6(1)$ & $3(1)$ \\
$\mathrm{F}(1)$ & $98(2)$ & $58(1)$ & $34(1)$ & $21(1)$ & $14(1)$ & $32(1)$ \\
$\mathrm{F}(2)$ & $69(2)$ & $52(1)$ & $34(1)$ & $-3(1)$ & $3(1)$ & $-10(1)$ \\
$\mathrm{F}(3)$ & $45(1)$ & $91(2)$ & $35(1)$ & $6(1)$ & $19(1)$ & $7(1)$ \\
\hline & & & & & & \\
\end{tabular}




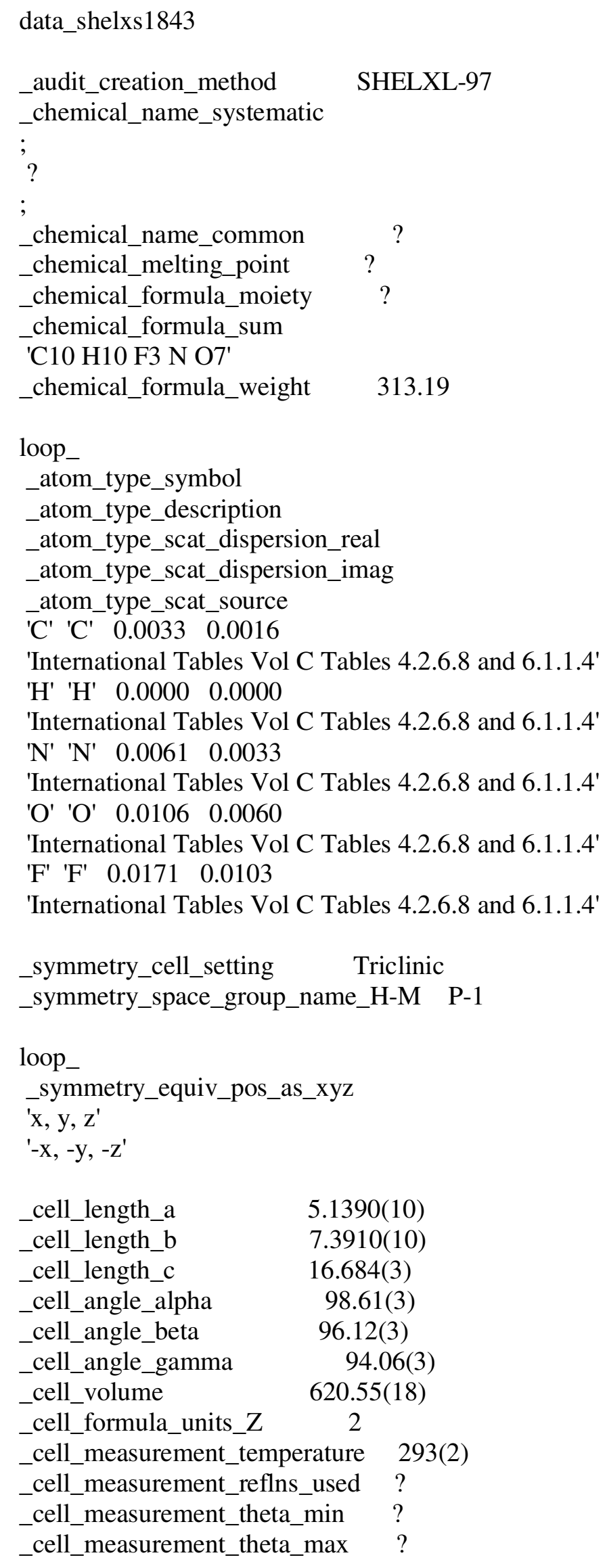




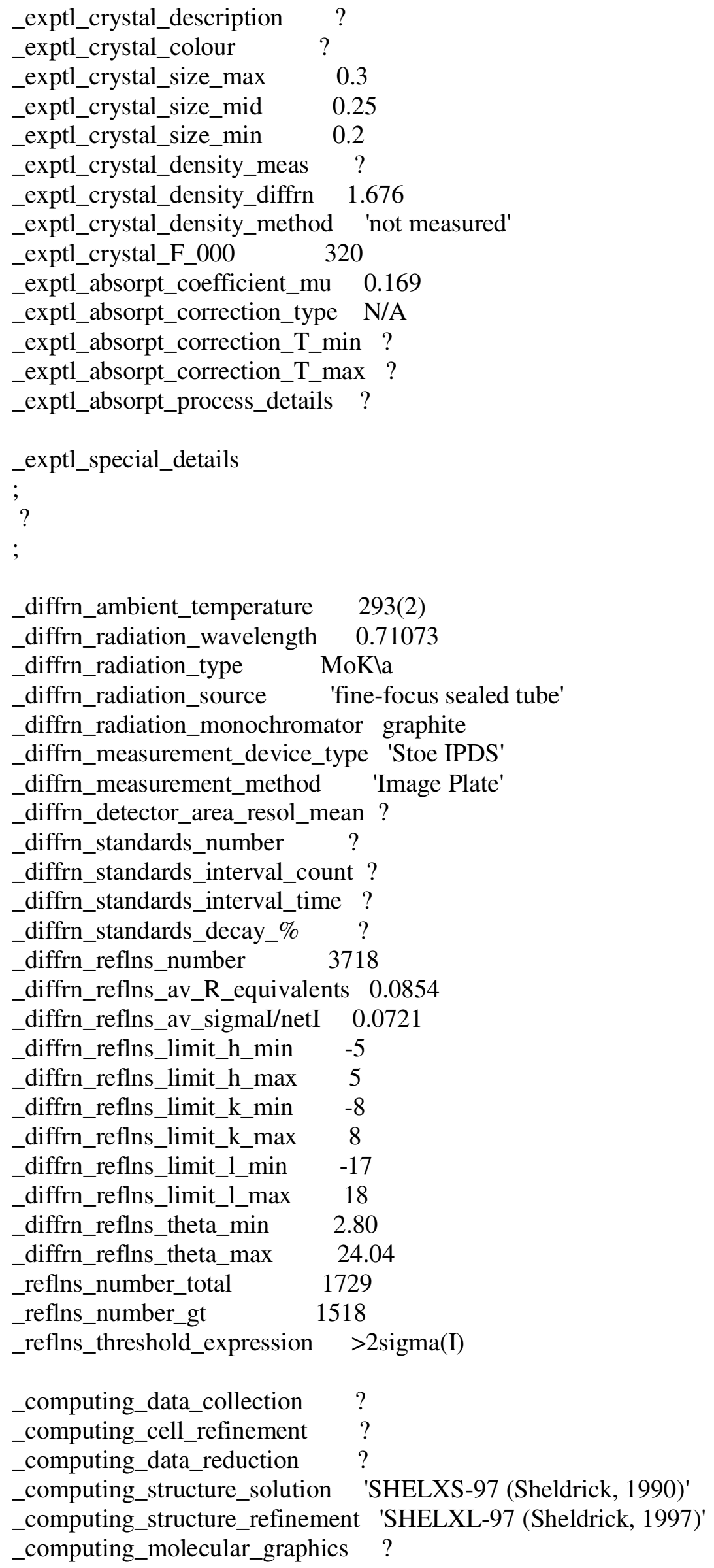


_computing_publication_material ?

_refine_special_details

;

Refinement of $\mathrm{F}^{\wedge} 2^{\wedge}$ against ALL reflections. The weighted R-factor $\mathrm{wR}$ and goodness of fit $\mathrm{S}$ are based on $\mathrm{F}^{\wedge} 2^{\wedge}$, conventional R-factors $\mathrm{R}$ are based on $\mathrm{F}$, with $\mathrm{F}$ set to zero for negative $\mathrm{F}^{\wedge} 2^{\wedge}$. The threshold expression of $\mathrm{F}^{\wedge} 2^{\wedge}>2 \operatorname{sigma}\left(\mathrm{F}^{\wedge} 2^{\wedge}\right)$ is used only for calculating $\mathrm{R}$-factors $(\mathrm{gt})$ etc. and is not relevant to the choice of reflections for refinement. R-factors based on $\mathrm{F}^{\wedge} 2^{\wedge}$ are statistically about twice as large as those based on $\mathrm{F}$, and Rfactors based on ALL data will be even larger.

_refine_ls_structure_factor_coef Fsqd

_refine_ls_matrix_type full

_refine_ls_weighting_scheme calc

_refine_ls_weighting_details

'calc $\mathrm{w}=1 /\left[\backslash \mathrm{s}^{\wedge} 2^{\wedge}\left(\mathrm{Fo}^{\wedge} 2^{\wedge}\right)+(0.0844 \mathrm{P})^{\wedge} 2^{\wedge}+0.4609 \mathrm{P}\right]$ where $\mathrm{P}=\left(\mathrm{Fo}^{\wedge} 2^{\wedge}+2 \mathrm{Fc}^{\wedge} 2^{\wedge}\right) / 3^{\prime}$

_atom_sites_solution_primary direct

_atom_sites_solution_secondary difmap

_atom_sites_solution_hydrogens geom

_refine_ls_hydrogen_treatment mixed

_refine_ls_extinction_method none

_refine_ls_extinction_coef ?

_refine_ls_number_reflns 1729

_refine_ls_number_parameters 226

_refine_ls_number_restraints 0

_refine_ls_R_factor_all 0.0583

_refine_ls_R_factor_gt 0.0529

_refine_ls_wR_factor_ref 0.1511

_refine_ls_wR_factor_gt $\quad 0.1465$

_refine_ls_goodness_of_fit_ref 1.048

_refine_ls_restrained_S_all 1.048

_refine_ls_shift/su_max $\quad 0.009$

_refine_ls_shift/su_mean 0.000

loop_

_atom_site_label

_atom_site_type_symbol

_atom_site_fract_x

_atom_site_fract_y

_atom_site_fract_z

_atom_site_U_iso_or_equiv

_atom_site_adp_type

_atom_site_occupancy

_atom_site_symmetry_multiplicity

_atom_site_calc_flag

_atom_site_refinement_flags

_atom_site_disorder_assembly

_atom_site_disorder_group

O1 O -0.0272(4) 0.1394(3) 0.28028(11) 0.0340(5) Uani $11 \mathrm{~d} \ldots$

O2 O 0.3784(4) 0.0639(3) 0.31758(12) 0.0383(6) Uani $11 \mathrm{~d} \ldots$

O3 O 0.7293(4) -0.4372(3) 0.10507(11) 0.0311(5) Uani $11 \mathrm{~d} \ldots$

O4 O 0.6006(4) -0.6422(2) 0.18391(12) 0.0325(5) Uani $11 \mathrm{~d} \ldots$ 
H4 H $0.7255-0.69500 .16830 .049$ Uiso 11 calc R . .

O5 O 0.8190(4) -0.2902(3) 0.29925(11) 0.0319(5) Uani $11 \mathrm{~d} \ldots$

O6 O 0.0040(4) 0.1933(3) 0.11420(11) 0.0351(6) Uani $11 \mathrm{~d} \ldots$

O7 O 0.2613(4) -0.0053(3) 0.05485(11) 0.0324(5) Uani $11 \mathrm{~d} \ldots$

N1 N 0.3762(5) -0.3552(3) 0.27309(13) 0.0237(6) Uani $11 \mathrm{~d} \ldots$

H1 H 0.234(7) -0.348(4) 0.290(2) 0.030(9) Uiso $11 \mathrm{~d} \ldots$

C1 C 0.1582(5) -0.0614(3) 0.18229(15) 0.0216(6) Uani $11 \mathrm{~d} \ldots$

H1A H 0.010(6) -0.140(4) 0.1779(16) 0.022(7) Uiso $11 \mathrm{~d} \ldots$

C2 C 0.1267(6) 0.0590(4) 0.11585(15) 0.0255(6) Uani $11 \mathrm{~d}$. . .

C3 C 0.3731(6) -0.1783(4) 0.06770(17) 0.0296(7) Uani $11 \mathrm{~d} .$.

H3A H 0.548(7) -0.178(4) 0.044(2) 0.039(9) Uiso $11 \mathrm{~d} \ldots$.

H3B H 0.251(6) -0.280(4) 0.0367(18) 0.027(7) Uiso $11 \mathrm{~d} \ldots$

C4 C 0.3883(5) -0.1729(3) 0.16038(15) 0.0221(6) Uani $11 \mathrm{~d} \ldots$

H4 H 0.550(7) -0.097(4) 0.1865(18) 0.031(8) Uiso $11 \mathrm{~d} \ldots$

C5 C 0.3712(5) -0.3691(4) 0.18442(15) 0.0218(6) Uani $11 \mathrm{~d}$. . .

H5 H 0.224(6) -0.437(4) 0.1615(16) 0.019(7) Uiso $11 \mathrm{~d} \ldots$

C6 C 0.5895(5) -0.4843(3) 0.15373(15) 0.0238(6) Uani $11 \mathrm{~d} \ldots$

C7 C 0.1884(6) 0.0547(4) 0.26807(16) 0.0265(7) Uani $11 \mathrm{~d}$. . .

C8 C -0.0287(9) 0.2489(5) 0.3614(2) 0.0421(9) Uani $11 \mathrm{~d} \ldots$

H8A H -0.193(11) 0.285(7) 0.366(3) 0.081(16) Uiso $11 \mathrm{~d} \ldots$

H8B H 0.092(10) 0.353(7) 0.373(3) 0.071(13) Uiso $11 \mathrm{~d} \ldots$

H8C H -0.010(8) 0.176(6) 0.402(3) 0.058(11) Uiso $11 \mathrm{~d} \ldots$

C9 C 0.6010(6) -0.3058(3) 0.32205(16) 0.0248(6) Uani $11 \mathrm{~d} \ldots$.

C10 C 0.5794(6) -0.2720(4) 0.41443(17) 0.0330(7) Uani $11 \mathrm{~d} \ldots$

F1 F 0.6821(5) -0.4075(3) 0.44793(11) 0.0603(7) Uani $11 \mathrm{~d} .$.

F2 F 0.7117(4) -0.1113(3) 0.45121(11) 0.0536(6) Uani $11 \mathrm{~d} \ldots$

F3 F 0.3328(4) -0.2696(3) 0.43166(11) 0.0563(6) Uani $11 \mathrm{~d}$. . .

loop_

_atom_site_aniso_label

_atom_site_aniso_U_11

_atom_site_aniso_U_22

_atom_site_aniso_U_33

_atom_site_aniso_U_23

_atom_site_aniso_U_13

_atom_site_aniso_U_12

O1 $0.0366(13) 0.0378(11) 0.0279(11)-0.0008(8) 0.0067(9) 0.0145(9)$

O2 0.0350 (14) $0.0431(13) 0.0331(12)-0.0018(9)-0.0041(9) 0.0084(10)$

O3 0.0324(13) 0.0318(11) 0.0328(11) 0.0086(8) 0.0122(8) 0.0080(9)

O4 0.0334(13) 0.0246(10) 0.0435(12) 0.0109(8) 0.0103(9) 0.0098(9)

O5 0.0238(12) 0.0423(12) 0.0309(10) 0.0092(8) 0.0044(8) 0.0020(9)

O6 0.0431(14) 0.0342(12) 0.0320(11) 0.0099(9) 0.0064(9) 0.0180(10)

O7 0.0429(14) 0.0331(11) 0.0268(10) 0.0126(8) 0.0102(8) 0.0155(9)

N1 0.0182(14) 0.0261(12) 0.0298(12) 0.0097(9) 0.0080(10) 0.0036(10)

C1 0.0213(15) 0.0197(13) 0.0240(13) 0.0048(10) 0.0031(10) 0.0005(11)

C2 0.0267(16) 0.0249(14) 0.0249(13) 0.0034(11) 0.0021(11) 0.0043(12)

C3 0.0360(19) 0.0283(15) 0.0290(15) 0.0096(11) 0.0088(12) 0.0156(13)

C4 0.0209(16) 0.0223(13) 0.0247(13) 0.0061(10) 0.0051(10) 0.0041(11)

C5 0.0170(15) 0.0220(13) 0.0267(13) 0.0055(10) 0.0025(10) 0.0003(11)

C6 0.0212(15) 0.0223(13) 0.0263(13) 0.0007(10) -0.0001(11) 0.0022(11)

C7 0.0299(18) 0.0232(14) 0.0271(15) 0.0052(11) 0.0058(12) 0.0010(12)

C8 0.051(2) 0.041(2) 0.0323(17) -0.0064(15) 0.0118(15) 0.0049(18)

C9 0.0266(17) 0.0231(13) 0.0268(14) 0.0075(10) 0.0064(11) 0.0037(11)

C10 0.0375(19) 0.0326(16) 0.0301(15) 0.0077(12) 0.0059(12) 0.0028(13) 
F1 0.0984(19) 0.0581(13) 0.0339(10) 0.0213(9) 0.0142(10) 0.0320(12)

F2 0.0693(15) 0.0519(12) 0.0336(10) -0.0034(8) 0.0032(9) $-0.0101(10)$

F3 0.0450(13) 0.0909(16) 0.0350(10) 0.0061(10) 0.0189(9) 0.0074(11)

_geom_special_details

;

All esds (except the esd in the dihedral angle between two 1.s. planes) are estimated using the full covariance matrix. The cell esds are taken into account individually in the estimation of esds in distances, angles and torsion angles; correlations between esds in cell parameters are only used when they are defined by crystal symmetry. An approximate (isotropic) treatment of cell esds is used for estimating esds involving l.s. planes.

;

loop_

_geom_bond_atom_site_label_1

_geom_bond_atom_site_label_2

_geom_bond_distance

_geom_bond_site_symmetry_2

_geom_bond_publ_flag

O1 C7 1.330(4) . ?

O1 C8 1.470(4) . ?

$\mathrm{O} 2 \mathrm{C} 7$ 1.201(4) . ?

O3 C6 1.212(3) . ?

O4 C6 1.341(3) . ?

O5 C9 1.224(3) . ?

O6 C2 1.215(3).?

O7 C2 1.339(3). ?

O7 C3 1.473(3) . ?

N1 C9 1.336(4) . ?

N1 C5 1.465(3). ?

$\mathrm{C} 1 \mathrm{C} 2$ 1.525(4). ?

C1 C4 1.533(4). ?

C1 C7 1.541(4) . ?

C3 C4 1.534(4) . ?

C4 C5 1.561(4) . ?

C5 C6 1.540(4) . ?

C9 C10 1.541(4) . ?

C10 F3 1.330(4) . ?

C10 F1 1.332(4) . ?

C10 F2 1.352(4) . ?

loop_

_geom_angle_atom_site_label_1

_geom_angle_atom_site_label_2

_geom_angle_atom_site_label_3

_geom_angle

_geom_angle_site_symmetry_1

_geom_angle_site_symmetry_3

_geom_angle_publ_flag

C7 O1 C8 116.2(3) .. ?

C2 O7 C3 111.9(2) . ?

C9 N1 C5 120.4(2) . . ?

C2 C1 C4 103.8(2). . ? 
C2 C1 C7 111.3(2)..?

C4 C1 C7 116.7(2) . . ?

O6 C2 O7 122.2(2) . ? ?

O6 C2 C1 128.8(2) . . ?

O7 C2 C1 109.0(2) . . ?

O7 C3 C4 103.5(2) . . ?

C1 C4 C3 102.4(2) .. ?

C1 C4 C5 114.1(2) .. ?

C3 C4 C5 112.3(2) .. ?

N1 C5 C6 110.8(2) . . ?

N1 C5 C4 109.8(2). . ?

C6 C5 C4 112.7(2) . . ?

O3 C6 O4 124.4(3) ..?

O3 C6 C5 122.4(2) . . ?

O4 C6 C5 113.2(2) . . ?

O2 C7 O1 124.8(2) .. ?

O2 C7 C1 124.5(3) . . ?

O1 C7 C1 110.6(2). . ?

O5 C9 N1 125.2(2) . . ?

O5 C9 C10 118.5(2) . . ?

N1 C9 C10 116.2(2) . . ?

F3 C10 F1 106.9(2) . . ?

F3 C10 F2 107.8(2) .. ?

F1 C10 F2 108.3(3) .. ?

F3 C10 C9 113.2(2) . . ?

F1 C10 C9 108.9(2) .. ?

F2 C10 C9 111.5(2) . . ?

_diffrn_measured_fraction_theta_max 0.885

_diffrn_reflns_theta_full 24.04

_diffrn_measured_fraction_theta_full 0.885

_refine_diff_density_max 0.288

_refine_diff_density_min -0.261

_refine_diff_density_rms 0.071 